\section{Dificultades en el diagnóstico de enteritis lúpica: presentación de un caso}

\author{
NORMA LIZETH ZÚÑIGA Z. a, MARÍA TERESA GONZALES J. \\ GONZALO LÓPEZ A. ${ }^{1,2}$, ALFREDO BRIONES ${ }^{1}$
}

\section{Lupus enteritis. Report of one case}

In lupus enteritis, circulating pathological immune complexes and thrombosis of intestinal vessels may occur, resulting in acute abdominal pain. We report a 24-year-old woman without a history of systemic lupus erythematosus (SLE), admitted for abdominal pain. An exploratory laparotomy found an appendicitis along with ascites. An appendectomy was performed, and the patient was discharged from the hospital two days later. Three days after discharge, the patient was admitted to another hospital due to the persistence of abdominal pain. An abdominal computed tomography scan showed diffuse mesenteric congestion, concentric bowel loops (double halo or target sign) and the presence of free fluid in the peritoneal cavity. Suspecting a rheumatic disorder, the diagnosis of SLE was confirmed by immunological studies. The patient was treated with pulses of methylprednisolone with good results.

(Rev Med Chile 2019; 147: 1073-1077)

Key words: Acute, Abdomen; Enteritis; Lupus Erythematosus, Systemic.
${ }^{1}$ Facultad de Medicina Humana de la Universidad Autónoma de Chiapas (UNACH), Tuxtla Gutiérrez. Chiapas, México. 2Departamento de Cirugía, Centro Médico "Jesús Gilberto Gómez Maza", Tuxtla Gutiérrez. Chiapas, México.

${ }^{a}$ Médico pasante en servicio social, Facultad de Medicina Humana de la Universidad Autónoma de Chiapas, Tuxtla Gutiérrez. Chiapas, México.

Trabajo no recibió financiamiento. Los autores declaran no tener conflictos de interés.

Recibido el 16 de noviembre de 2018, aceptado el 9 de septiembre de 2019.

Correspondencia a:

Dr. Alfredo Briones A Facultad de Medicina Humana de la Universidad Autónoma de Chiapas (UNACH), Tuxtla Gutiérrez. Chiapas, México. alfred725@hotmail.com
E 1 lupus eritematoso sistémico (LES) es una enfermedad autoinmune de curso crónico, de causa desconocida, con diversas manifestaciones clínicas; se caracteriza por una respuesta inflamatoria mediada por la formación de múltiples inmunocomplejos que pueden depositarse en varios órganos y tejidos ${ }^{1}$.

Se estima que al menos 5 millones de personas en todo el mundo tienen LES, y más de 100.000 casos nuevos son diagnosticados cada año ${ }^{2}$, en México se ha proyectado una prevalencia de $0,06 \%{ }^{3}$.

La afectación del tracto gastrointestinal es relativamente frecuente en los pacientes con LES, con una frecuencia de $40 \%$ en los casos de enfermedad activa. Las manifestaciones clínicas varían desde dolor abdominal inespecífico, hasta un cuadro de abdomen agudo (AA) llamado vasculitis mesentérica o enteritis lúpica $(\mathrm{EL})^{4}$, siendo este cuadro clínico la causa más frecuente de consulta por trastornos abdominales agudos, en donde $10 \%$ del AA en el ámbito hospitalario y $5 \%$ en la consulta de atención primaria pueden requerir cirugía, la morbilidad-mortalidad de esta condición clínica es alta y se relaciona con la demora entre el inicio de los síntomas y la solución del problema ${ }^{5}$. No obstante, continúan siendo excepcionales los casos reportados de EL como manifestación inicial en donde se desconozca el diagnóstico de LES'.

Por todo lo anterior, el principal objetivo de la presente investigación fue analizar un caso clínico de EL que cursó inicialmente con una aparente apendicitis, en un paciente sin antecedentes de LES, con la finalidad de analizar las dificultades en el diagnóstico y poder coadyuvar en la integración expedita de una estrategia clínica para el tratamiento oportuno de esta patología.

\section{Caso clínico}

Presentamos el caso de una mujer de 24 años que ingresó a un primer hospital por referir eva- 
cuaciones diarreicas de un mes de evolución y dolor agudo tipo cólico en epigastrio con irradiación difusa; en consecuencia, bajo el diagnóstico de AA, a esta paciente se le realizó una laparotomía exploratoria (LAPE), encontrándose una posible apendicitis en fase II y líquido de ascitis en cavidad por lo que se realizó una apendicectomía y se drenó el líquido. En esta intervención se tomó una muestra histológica de epiplón, peritoneo, apéndice cecal y líquido de ascitis para su posterior análisis. Posteriormente, la paciente egresó de ese hospital.

Tres días después, la paciente es ingresada a un segundo hospital por referir persistencia de dolor abdominal difuso. En la exploración abdominal, se observó una herida quirúrgica con una adecuada cicatrización, a la palpación se encontró un abdomen blando, depresible, con dolor a la presión en ambas fosas iliacas.

Los exámenes mostaron: leucocitosis de 8.710/ $\mathrm{mm}^{3}$, linfopenia en $5,6 \%$ y un recuento absoluto de $0,49 \times 10^{\wedge} 3 / \mu \mathrm{l}$. Mientras que los tiempos de coagulación, la química sanguínea, el perfil hepático, la gasometría arterial y el examen general de orina no evidenciaron alteraciones. En la ecografía se reportaron datos ultrasonográficos característicos de pielectasia renal bilateral y líquido libre en cavidad.

En consideración a todo lo anterior, se instalo sonda nasogástrica a derivación y se indicó ayuno, con una vigilancia continua. En el cuarto día de evolución, la paciente mostró cierta mejoría al cuadro previo. Los resultados del estudio histopatológico inicial reportaron: epiplón con presencia de congestión vascular leve y peritoneo con infiltrado inflamatorio crónico, apéndice cecal y líquido de ascitis con estudio cito-químico normal. Posteriormente, se continuó estudiando a la paciente mediante la realización de pruebas de función hepática, gasometría arterial, panel viral, Western blot, urocultivo y marcadores tumorales, cuyos resultados se encontraron dentro de la normalidad.

Se solicitó una tomografía axial computada (TAC) abdominopélvica, que mostró una cantidad moderada de líquido en cavidad con predominio en el hueco pélvico; congestión mesentérica difusa y una disposición de las asas intestinales en forma centralizada, posiblemente asociada a líquido libre (signo de la diana) (Figura 1). Estos hallazgos fueron interpretados como una aparente enteritis bacteriana, condición que favoreció la solicitud de una punción abdominal para la obtención de líquido peritoneal y la realización del cultivo correspondiente, el cual tuvo un reporte negativo. $\mathrm{La}$ antibioticoterapia empírica previamente indicada con base en vancomicina fue suspendida.

Después, se solicitó una valoración por gastroenterología; la paciente refirió antecedentes de depresión mayor a los 13 años, posible fenómeno de Raynaud y caída de cabello desde la adolescencia. En consecuencia, se empezó a inferir un probable diagnóstico de enfermedad reumatológica y se solicitaron estudios inmunológicos para confirmar el diagnóstico. Se obtuvieron los siguientes resultados: ANA (+) 11,80 (positivo $>1,50$ ), C3 24,6 mg/dL (valor de referencia: $70-180 \mathrm{mg} / \mathrm{dL}$ ), C4 2,6 mg/dL (valor de referencia: $16-45 \mathrm{mg} / \mathrm{dL}$ ), anti-Sm 3,58 ratio (positivo $>1,10$ ) y el marcador anti-dsDNA (-).

Finalmente, confirmado el diagnóstico de EL se iniciaron pulsos con metilprednisolona por vía intravenosa $(1 \mathrm{~g} / 1 \mathrm{~h})$, esta estrategia terapéutica favoreció el egreso de la paciente por una mejoría clínica.

\section{Discusión}

La EL, cuándo se enmascara con un cuadro de apendicitis en los pacientes sin antecedentes de LES, se convierte en un verdadero reto para la integración diagnóstica, probablemente debido a lo heterogéneo de los síntomas y a la infrecuencia con la que la EL es la primera manifestación de LES.

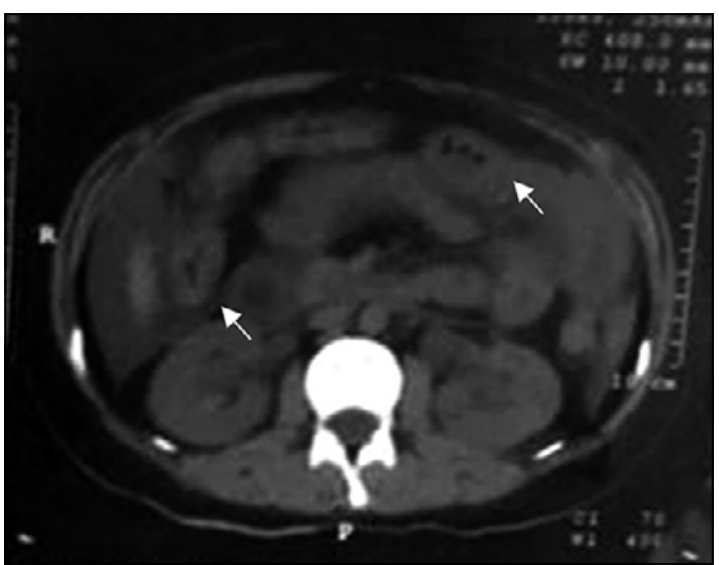

Figura 1. En la tomografía axial computarizada de abdomen se observa signo de la diana (flechas). 
El dolor abdominal fue el síntoma común de los casos revisados, incluyendo el del presente reporte. Sin embargo, llama la atención que en todos los casos, el dolor referido tuvo características variantes, como la localización difusa, los diferentes grados de intensidad y la presencia o ausencia de irradiación. No obstante, el dolor abdominal tuvo una evolución mayor a 72 h en la mayoría de los casos analizados (Tabla 1$)^{6-9}$.

En otro contexto, estudios previos han informado la variabilidad en los resultados de la biometría que no guardan un patrón contante asociado con la EL. Por ejemplo, 52\% de casos de LES presentó hemoglobina baja, en $40 \%$ existieron

Tabla 1. Comparación de 5 casos reportados con enteritis lúpica como primera manifestación de Lupus eritematoso sistémico

\begin{tabular}{|c|c|c|c|c|c|c|c|c|}
\hline & Sexo & Edad & $\begin{array}{c}\text { Cirugía } \\
\text { abdominal } \\
\text { previa }\end{array}$ & $\begin{array}{l}\text { Días } \\
\text { de } \\
\text { evolución }\end{array}$ & Síntomas & Laboratorios & TAC & $\begin{array}{l}\text { Estudios } \\
\text { inmuno- } \\
\text { lógicos }\end{array}$ \\
\hline $\begin{array}{l}\text { Barrera y } \\
\text { cols. }{ }^{7}\end{array}$ & $\mathrm{~F}$ & 33 & No & 60 & $\begin{array}{l}\text { - Anorexia } \\
\text { - Fiebre } \\
\text { - Caída de } \\
\text { cabello } \\
\text { - Poliartralgia } \\
\text { - Dolor } \\
\text { abdominal }\end{array}$ & - & - & $\begin{array}{l}\text { Anti-ANA: }(+) \\
\text { Anti-RNP: }(+) \\
\text { C3: bajo } \\
\text { C4: bajo }\end{array}$ \\
\hline $\begin{array}{l}\text { Vallejo y } \\
\text { cols. }^{8}\end{array}$ & $\mathrm{~F}$ & 16 & No & 11 & $\begin{array}{l}\text { - Dolor } \\
\text { abdominal } \\
\text { - Náuseas } \\
\text { - Malestar } \\
\text { general } \\
\text { - Fiebre }\end{array}$ & 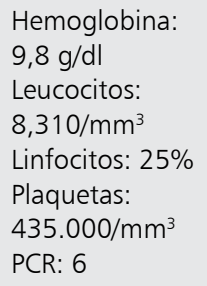 & - & $\begin{array}{l}\text { Anti-ANA: }(+) \\
1 / 360 \text { patrón } \\
\text { moteado } \\
\text { C3: } 39 \mathrm{mg} / \mathrm{dL} \\
\text { C4: } 9,1 \mathrm{mg} / \mathrm{d}\end{array}$ \\
\hline $\begin{array}{l}\text { Solis y } \\
\text { cols. }{ }^{9}\end{array}$ & M & 24 & No & 3 & $\begin{array}{l}\text { - Dolor } \\
\text { abdominal }\end{array}$ & - & - & - \\
\hline $\begin{array}{l}\text { De Zubiría } \\
\text { y cols. }{ }^{10}\end{array}$ & $\mathrm{~F}$ & 24 & Sí & 4 & $\begin{array}{l}\text { - Dolor } \\
\text { abdominal } \\
\text { - Náuseas } \\
\text { - Vómitos }\end{array}$ & - & $\begin{array}{l}\text { - Líquido libre en } \\
\text { cavidad abdomi- } \\
\text { nal } \\
\text { - Engrosamiento } \\
\text { difuso de las } \\
\text { asas intestinales } \\
\text { - Hepatomegalia } \\
\text { leve } \\
\text { - Hidronefrosis }\end{array}$ & $\begin{array}{l}\text { Anti-ANA: }(+) \\
\text { 1/640 patrón } \\
\text { moteado } \\
\text { Anti-DNA: (+) } \\
\text { Anti-Ro: (+) } \\
\text { Anti-LA: (+) } \\
\text { Anti-SM: (+) } \\
\text { Anti-RNP: (+) } \\
\text { C3: } 16 \mathrm{mg} / \\
\text { dLC4: } 21 \mathrm{mg} / \mathrm{dL}\end{array}$ \\
\hline $\begin{array}{l}\text { Presente } \\
\text { caso }\end{array}$ & $\mathrm{F}$ & 24 & Sí & 30 & $\begin{array}{l}\text { - Dolor } \\
\text { abdominal } \\
\text { - Diarrea } \\
\text { - Vómitos }\end{array}$ & $\begin{array}{l}\text { Hemoglobina: } \\
\text { 12,8 g/dL } \\
\text { Leucocitos: } \\
\text { 8,710/mm³ } \\
\text { Linfocitos: } \\
\text { 5,6\% RA 0,49 } \\
\text { x10^3/ul } \\
\text { Plaquetas: } \\
\text { 493x10^3/ul } \\
\text { PCR: } 0,7 \text { mg/dL }\end{array}$ & $\begin{array}{l}\text { - Congestión me- } \\
\text { sentérica difusa } \\
\text { - Líquido libre } \\
\text { que centraliza } \\
\text { asas intestinales }\end{array}$ & $\begin{array}{l}\text { Anti-ANA: }(+) \\
\text { Anti-DNA: }(-) \\
\text { Anti-SM: }(+) \\
\text { C3: } 24,6 \mathrm{mg} / \mathrm{dL} \\
\text { C4: } 2,6 \mathrm{mg} / \mathrm{dL}\end{array}$ \\
\hline
\end{tabular}

F: femenino; M: masculino; PCR: proteína C reactiva; Anti-ANA: anticuerpos antinucleares; Anti-DNA: anticuerpos de doble cadena; Anti-Ro: anticuerpos topoisomerasa Ro; Anti-LA: anticuerpos topoisomerasa LA; Anti-SM: anticuerpos Smith, Anti-RNP: anticuerpos anti-ribonucleoproteina. 
datos de linfopenia ${ }^{10} \mathrm{y}$ escasos estudios refirieron leucopenia ${ }^{11}$. Lo anterior está en concordancia con los resultados de linfopenia encontrados en el presente estudio y los datos de anemia reportados en un estudio previo ${ }^{7}$. Sin embargo, llama la atención que en la mayoría de los casos revisados, no se mostraron los resultados de la biometría hemática (Tabla 1$)^{6-9}$. Lo anterior sugiere la posibilidad de que alteraciones hemáticas asociadas a LES podrían pasar inadvertidas.

Por otra parte, los datos de serositis del peritoneo podrían representar dificultad en la sospecha clínica de LES, ya que esta inflamación representa un dato inespecífico para el diagnóstico de LES. No obstante, hubiera sido interesante la búsqueda de vasculitis en tejido gastrointestinal (GI) debido a que esta alteración se ha relacionado con LES en reportes previos ${ }^{12}$.

Con respecto a la utilidad de los estudios inmunológicos, en la revisión de casos previos se destaca un patrón constante para la presencia de anti-ANA + y los bajos niveles de complemento en los pacientes con EL. La importancia de solicitar la determinación de estos anticuerpos, en los pacientes bajo protocolo de estudio, radica en la posibilidad de evidenciar, no solo la presencia de la enfermedad, si no la actividad del LES.

Además, en la mayoría de los estudios revisados la TAC ha reportado la presencia de isquemia mesentérica asociada a una dilatación de la pared intestinal de forma focalizada o difusa, además del signo de la diana, asociado tanto a la presencia de ascitis, como a edema y vasodilatación mesentérica. Lo anterior, reafirma la importancia que tiene la TAC para el diagnóstico expedito y específico ${ }^{13}$. No obstante, una desventaja es la inespecificidad de estos signos para el diagnóstico de EL, debido a que imágenes de aparente isquemia intestinal podrían estar también presentes en pacientes con: pancreatitis, obstrucción intestinal mecánica, peritonitis o enfermedad inflamatoria intestinal ${ }^{14}$.

Es preocupante que en la totalidad de los estudios revisados, únicamente se indicara la TAC en los pacientes con antecedentes quirúrgicos como parte de la integración diagnóstica (Tabla $1)^{6-9}$. La falta de TAC en estos pacientes podría estar relacionada con la preocupación que tiene el cirujano en evitar complicaciones asociadas a perforación GI. Esta última premisa pareciera estar basada en la relación directa entre el aumento de la morbimortalidad en los pacientes con EL y el mayor período de tiempo trascurrido antes de la realización de la $\mathrm{LAPE}^{11,15}$. En contraste, otro estudio ha reportado que a pesar de la evolución del dolor, ninguno de los pacientes con EL tratados tempranamente con corticoides desarrolló una perforación $\mathrm{GI}^{11}$. Por lo anterior, se reafirma la importancia del requerimiento de la realización de la TAC en la totalidad de los pacientes que cursan con AA con una evolución mayor a $72 \mathrm{~h}$.

Finalmente, no está por demás reconocer la importancia que tiene la realización y análisis de una historia clínica completa, como hilo conductor fundamental en el diagnóstico de LES.

En conclusión, el diagnóstico de EL como primera manifestación de LES requiere de un análisis más puntualizado de las variables clínicas, lo que abonaría a una mayor sospecha clínica y fundamentaría el requerimiento tanto para la realización de la TAC como de los estudios inmunológicos. Todo esto con la finalidad de favorecer un diagnóstico temprano de EL y evitar una LAPE innecesaria.

\section{Referencias}

1. La Paglia GMC, Leone MC, Lepri G, Vagelli R, Valentini E, Alunno A, et al. One year in review 2017: systemic lupus erythematosus. Clin Exp Rheumatol 2017; 35 (4): 551-61.

2. Hermansen ML, Lindhardsen J, Torp-Pedersen C, Faurschou M, Jacobsen S. Incidence of Systemic Lupus Erythematosus and Lupus Nephritis in Denmark: A Nationwide Cohort Study. J Rheumatol 2016; 43 (7): 1335-9.

3. Peláez-Ballestas I, Sanin L, Moreno-Montoya J, Álvarez-Nemegyei J, Burgos-Vargas R, Garza-Elizondo M. Epidemiology of the rheumatic diseases in Mexico A Study of 5 regions based on the COPCORD methodology. J Rheumatol Suppl 2011; 86: 3-8.

4. Katsanos KH, Voulgari PV, Tsianos EV. Inflammatory bowel disease and lupus: a systematic review of the literature. J Crohns Colitis 2012; 6 (7): 735-42.

5. Zavaleta JA, Vázquez JH. Síndrome de abdomen agudo. En: Morales JL. Tratado de cirugía general. Ciudad de México (México): Editorial el Manual Moderno; 2017, pp. 975.

6. Barrera A, Bannura C, Correidora S, Garcia R. Apendicitis aguda secundaria a una vasculitis lúpica. Rev Chil Cir 2002; 54 (5): 529-31.

7. Vallejo FJ, Medina LF. Dolor abdominal agudo Un 
enfoque clínico de la vasculitis lúpica abdominal. Acta Med Colomb 2007; 32 (1): 25-8.

8. Solís N, Rojas R, Mora F. Masculino 24 años con vasculitis intestinal. Reporte de caso y revisión de la literatura. Revista clínica de la escuela de medicina UCR-HSJD 2013; 3 (5): 7-10.

9. De Zubiría A, Calderón M, Calderón W, Patiño A, Echeverry T, de la Cruz G. Pesudo-obstrucción lúpica Reto diagnóstico. Acta Med Colomb 2012; 37 (4): 207-10.

10. Janssens P, Arnaud L, Galicier L, Mathian A, Hie M, Sene D, et al. Lupus enteritis: from clinical findings to therapeutic management. Orphanet J Rare Dis 2013; 3: 8-67.

11. Lee CK, Ahn MS, Lee EY, Shin JH, Cho YS, Ha HK, et al. Acute abdominal pain in systemic lupus erythematosus: focus on lupus enteritis (gastrointestinal vasculitis). Ann Rheum Dis 2002; 61 (6): 547-50.

12. Peña R, Almanza E, Vargas E, Zaragoza GR, Espinosa $\mathrm{P}$, Castañeda RA, et al. Gastrointestinal vasculitis in an adolescent with systemic lupus erythematosus and catastrophic antiphospholipid syndrome. Endoscopia. 2016; 28 (4): 174-7.

13. Taviloglu K. When to operate on abdominal stab wounds. Scand J Surg 2002; 91 (1): 58-61.

14. Taourel PG, Deneuville M, Pradel JA, Régent D, Bruel JM. Acute mesenteric ischemia: diagnosis with contrast-enhanced CT. Radiology 1996; 199 (3): 632-6.

15. Koh ET, Boey ML, Feng PH. Acute surgical abdomen in systemic lupus erythematosus-an analysis of 10 cases. Ann Acad Med Singapore 1992; 21 (6): 833-7. 